\title{
The clinical case of an oncological patient affected by mandibular osteonecrosis and literature review about therapeutic alternatives
}

\author{
Francesca Marasciulo ${ }^{*}$, Giuseppe Lomurno ${ }^{2}$, Mario Mandalà ${ }^{1}$ and Fausto Roila ${ }^{1}$ \\ ${ }^{1}$ Struttura Complessa di Oncologia Medica, Azienda Ospedaliera - Universitaria Santa Maria della Misericordia, Perugia, Italy \\ ${ }^{2}$ Struttura Semplice Dipartimentale di Odontoiatria Chirurgica e Ambulatoriale, Azienda Ospedaliera - Universitaria Santa Maria della Misericordia, Perugia, Italy
}

\begin{abstract}
Mandibular osteonecrosis is a rare but important side effect of the use of radiotherapy, bisphosphonates, RANK ligand inhibitors and other antiangiogenics; it is crucial to implement prevention, perform an adequate differential diagnosis and quickly set up a therapy in order to avoid the development of complications. We describe the clinical case of a 51-year-old patient, the history of the disease and the treatment carried out.
\end{abstract}

\section{Introduction}

Mandibular osteonecrosis is an infrequent complication but with a gradual increasing incidence (phenomenon linked to the increase in neoplastic diseases, the higher survival rate of patients and induced by antineoplastic treatment) related to the use of antiresorptive drugs (especially zoledronic acid), RANK ligand inhibitors (i.e., denosumab) and/or antiangiogenics (i.e., bevacizumab), particularly if administered intravenously $[1,2]$. It is not excluded that one of the triggering causes may be poor bone turnover and remodeling, insufficient blood supply and constant microtraumas; necrosis can occur in an exposed area of the bone or through a fistula in correspondence with the maxillofacial region [3]. In most cases the mandibular bone is involved (68\%), in a lower percentage the foci are localized on the maxillary bone $(28 \%)$, persisting for eight weeks or more, during or following medical therapy with the aforementioned drugs and after excluding the use of radiotherapy on the affected area and the presence of local metastases. It is of fundamental importance to adopt prevention strategies, since in most cases there is local trauma at the base of the complications, and, if the infection is advanced, promptly to intervene with surgery and massive concomitant antibiotic therapy [2].

\section{Clinical case}

A 51-year-old patient came to our attention in the year 2000, after performing in July the surgery of supero-external quadrantectomy of the left breast and axillary lymphadenectomy following the diagnosis of infiltrating ductal carcinoma G1, $12 \mathrm{~mm}$, ER $60 \%$, PgR $<5 \%$, Ki67 15\%, c-erb-B2 negative, with micrometastases in $1 / 15$ axillary lymph nodes (pT1cN1M0). In August 2000 she began adjuvant chemotherapy according to the EC scheme (epirubicin and cyclophosphamide) for four cycles, followed by another four cycles of CMF (cyclophosphamide, methotrexate, 5-fluorouracil). In addition, she was submitted to adjuvant radiotherapy on the same breast and adjuvant hormone therapy with tamoxifen and LHRH analogue. In January 2003 tamoxifen was replaced with anastrozole and leuprorelin due to the iatrogenic increase in the thickness of the endometrium. The treatment continued until July 2006.
Subsequent follow-up was negative for disease progression until 2016, when, due to the appearance of an irritating cough and variation in the timbre of the voice, a series of tests were performed, including a chest-abdomen CT which highlighted the appearance of multiple pulmonary micro and macronodules and metastatic bone lesions in the sacral region and at the level of some lumbar metamers. Therefore a biopsy was carried out for in-depth diagnostic analysis of the sacral lesion, which allowed to make the diagnosis of breast carcinoma metastases. With regard to the disease characteristics, the patient's age and the treatments already carried out, it was decided to start chemotherapy with weekly paclitaxel plus the addiction of bisphosphonates, which determined a partial response of the disease.

In consideration of the onset of neurological toxicity characterized by paresthesia in the distal part of the upper limbs and nail toxicity related to paclitaxel, the latter was replaced with tamoxifen and LHRH analogue, continuing with bisphosphonates. In September 2019 the patient underwent tooth extraction and bone debridement for osteonecrosis of the right mandible following the prolonged use of zoledronic acid (from February 2017 to June 2019), and subsequent antibiotic therapy with amoxicillin $1 \mathrm{~g}$ /day for five days, diet soft and cold for a few days, applications of chlorhexidine gel on the operated area and ibuprofen $600 \mathrm{mg}$ three times a day for another three days, with clear clinical-radiological benefit. In March 2020 there was a relapse of osteonecrosis, treated with right mandibular sequestrectomy, amoxicillin sodium/potassium clavulanate $1 \mathrm{~g}$ three times a day,

*Correspondence to: Francesca Marasciulo, Complex Structure of Medical Oncology, University Hospital Santa Maria della Misericordia of Perugia, Piazzale Giorgio Menghini, 1, 06129 Perugia (PG), Italy, Tel: 3661765864; E-mail: fmara@myuax.com

Key words: antibiotic therapy, antiangiogenics, bisphosphonates, surgery, mandibular osteonecrosis, prevention

Received: June 16, 2021; Accepted: June 22, 2021; Published: June 25, 2021 
metronidazole $500 \mathrm{mg}$ twice a day for five days and paracetamol 1000 $\mathrm{mg}$ as needed in case of pain; the patient was advised to have a soft lukewarm diet for at least twenty days and careful oral hygiene with a soft-bristled toothbrush, with complete clinical resolution. Meanwhile, the patient continued hormone therapy and LHRH analogue, obtaining a stability of metastatic breast disease until the last follow-up in January 2021.

\section{Discussion and review of the literature}

Mandibular osteonecrosis is defined as a potentially serious side effect of drugs that modify bone metabolism and inhibit angiogenesis, generally manifesting as an infection of the jaw (mainly in the lingual area of the alveolar border of lower premolars and molars, site most exposed to mechanical trauma of chewing and reduced bone turnover with a high rate of remodeling) [3] in people of advanced age. Its incidence varies from approximately $1 \%$ to $10 \%$ of cases and, as major triggers have been identified for invasive dental procedures (especially in patients with a history of periodontal or periapical disease), injection of intravenous drugs, the duration, frequency of administration and their use in the context of multiple myeloma and metastatic breast cancer [1]. It is essential to make a differential diagnosis, especially in cases where the lesions are not clinically visible; in fact, periodontal disease, osteomyelitis, sinusitis, radiological osteonecrosis, primary bone neoplasms and metastases can cause the same symptoms as pharmacological mandibular osteonecrosis (pain, dental motility, gingival swelling, erythema and ulceration), that in about $30 \%$ of cases it is asymptomatic $[1,3]$.

Among others, it is important to consider the 2014 classification system based on clinical parameters drawn up by the American Association of Oral and Maxillofacial Surgeons in order to have a reference in the choice of the most appropriate therapy quantifying the severity and extent, which divides osteonecrosis into four stages in ascending order (stage 0: no clinical signs of osteonecrosis, inability to confirm the diagnosis; stage 1: exposure and necrosis or presence of a fistula, no symptoms or signs of infection; stage 2: previous stage plus signs of infection and/or symptoms; stage 3: exposure and necrosis or presence of a fistula, pain, infection plus at least one other of the symptoms, such as necrosis extending outside the alveolar region, pathological fracture, extraoral fistula, antral oral communication or oral nasal and/or osteolysis extending to the lower mandibular border or to the floor of the maxillary sinus) [1]. Although this system is still clinically functional today, it is not evidence-based, so staging should be performed based on the practitioner's clinical experience [3].

In accordance with the most important international guidelines it can be said that prevention in this area is of crucial importance (in fact, it may be more effective than standard therapy); it is recommended that all patients planning treatment with intravenous bisphosphonates perform a complete dental evaluation, including radiological examination of the oral cavity and dental visit, in order to evaluate the presence of dental elements susceptible to extractive surgery and to guarantee periodontal health to avoid the development of infections, also considering the need for subsequent dental follow-up approximately every six months during antineoplastic therapy [1].

As regards the treatment it is useful to underline the two fundamental strategies to be implemented in cases in which osteonecrosis is in the development phase or has already established, such as the continuation of cancer therapy and the guarantee of a good quality of life through the control of pain, local disease and medical education of the patient (performing adequate daily dental hygiene, treating local infections and diabetes, abolition of tobacco and alcohol, etc.) [3]. The goals of treatment in patients who have received a diagnosis of mandibular osteonecrosis can therefore be achieved through the use of analgesics, broad-spectrum antibiotics and, when necessary, conservative or radical surgery [1]. In addition, it is important to assess whether it is appropriate to continue or suspend osteoclasts inhibitors in the case of necrosis, but this decision will be weighted on each individual patient, based on age and comorbidities; in fact, the option of continuing therapy on a non-continuous basis is still being evaluated; if on one hand this modality would bring the benefit of stabilizing the infection and reducing the risk of spread to other sites, on the other hand it could cause the worsening of the underlying disease [3]. In any case it is advisable to adopt a multidisciplinary approach and establish a good communication with the patient, so that treatment adherence is also better [4]. Initially, it is suggested the use of antibiotics, which can be administered topically, orally or intravenously. Topical antibiotic therapy makes use of $0.12 \%$ chlorhexidine which is a bacteriostatic and bactericidal agent, used as a sole therapy in the initial stages of the disease and associated with other drugs in the later stages; the oral route of administration is the first choice, and considering that in most cases the infections are polymicrobial (characterized by colonization by Actinobacteria, Fusobacteria, Bacterioides), the most suitable drug would be a penicillin or alternatively clindamycin, fluoroquinolones and/or metronidazole, for a duration of two weeks in the early stages and four-six weeks in the more advanced stages. In cases resistant to the aforementioned therapy and in the absence of alternatives, it would be advisable to opt for an intravenous approach lasting about six weeks [5].

Although there is little evidence in this regard (currently controlled trials are not available), teriparatide, pentoxifylline, tocopherol, hyperbaric oxygen, low power laser therapy, platelet-rich plasma and topical medical ozone application have been considered as therapeutic strategies.

In particular, with regard to teriparatide (composed of 34 amino acids of the N-terminal chain of the parathyroid hormone, which maintains its anabolic effects, including bone remodeling) there are not yet sufficient studies to confirm its safety, the duration of its effectiveness on the control of osteonecrosis and the fact that this drug can stimulate cell proliferation is still being evaluated. Despite the comparison with placebo, it showed a superiority with respect to the resolution of the infection, so before administration individual benefitrisk ratio needs to be assessed [3]. The recommended dosage is 20 micrograms subcutaneously per day for approximately two years [2].

Pentoxifylline is a non-selective phosphodiesterase inhibitor that improves peripheral blood flow and induces the action of anti-TNFalpha, and tocopherol (vitamin E) contributes to the elimination of free radicals, both of which act by reducing inflammation, fibrosis and hence the risk of necrosis; although other studies are needed to define the optimal dose and duration of treatment. These two drugs in combination (slow-release pentoxifylline at a dose of $400 \mathrm{mg}$ twice daily and tocopherol at a dose of 1000 IU daily for thirty-six weeks) proven to be a valid alternative for treating mandibular osteonecrosis, with a good cost-benefit ratio [6].

Hyperbaric oxygen therapy has the function of providing a greater supply of oxygen to tissues with poor vascularization, ensuring optimal healing of the same and a greater bone turnover, for this reason it has long been used for the treatment of osteoradionecrosis, the evidence in favor of its use in the field of mandibular osteonecrosis is still scarce [2], and above all its benefit has been contextualized in a scheme of multimodal therapy, attributing the benefit especially to the concomitant use of surgery and antibiotic therapy. 
Another option to consider is the low power laser which helps to regulate metabolism, promote scarring and reduce pain and, when combined with high power lasers, could bring about complete mucosal healing and reduce microbial contamination; moreover, it has also been demonstrated an adjuvant role of the laser if used in combination with surgery, in fact it would improve the results.

Not to be underestimated, even with a preventive purpose, the use of plasma rich in platelets or platelet gel, which is an autologous source of growth factors and specific cytokines that stimulate angiogenesis, cell differentiation, the production of collagen and anti-inflammatory agents, thus promoting bone remodeling and soft tissue healing; in those patients who have not benefited from conservative therapies it could be useful to associate these derivatives with debridement or marginal osteotomy surgery, taking into account that new studies are needed to ensure in any case their effectiveness [6].

An effective, safe and simple alternative to perform is the topical insufflation of ozone, before or after conservative or surgical treatment. Ozone, which in this case is produced from pure oxygen, is a gas that has been shown to have antimicrobial and reparative-healing properties, thanks to its ability to increase the amount of red blood cells and hemoglobin, to stimulate leukocyte diapedesis and phagocytosis of the histiocytic reticular system. The procedure involves the administration of azithromycin $500 \mathrm{mg}$ per day in the ten days prior to treatment, in order to avoid the progress of the infectious process by reducing its extension, the dehiscence of the mucous membranes and to allow adequate oral hygiene to be maintained. Subsequently, the gas is applied at least ten times (once every three days) on each lesion until complete spontaneous expulsion of the necrotic bone and re-epithelialization of the mucosa [7].

Finally, surgery is one of the pillars in the definitive treatment of the late stages of mandibular osteonecrosis, in relation to the stage of the disease (and in general starting from the second) there are several more or less conservative approaches: curettage is based on the elimination of deposits that cause infection, sequestrectomy which consists in the removal of necrotic bone fragments, debridement with marginal resection which is the removal of the entire necrotic area, preserving the lower edge of the mandible, segmental resection which refers to the removal of the mandibular bone including its lower edge [5]. During the surgical act, the fluorescence induced by a tetracycline is generally used, an important guide for the operator; the technique involves the administration of doxycycline $100 \mathrm{mg}$ twice a day for the ten days prior to surgery, the fluorescence is monitored during the operation thanks to an ultraviolet light that emits the system and which, applied to the affected area, allows to distinguish necrotic bone (pale bluish-white color) from healthy (fluorescent) bone [2].

An innovative study has also shown that the implantation of a human amniotic membrane patch helps to improve the patient's quality of life, facilitating scarring and reducing the sensation of pain; it is a tissue obtained from the placenta rich in growth factors and tissue metalloprotease inhibitors, and poor in HLA-A, B, C or beta2 microglobulin antigens, which is why it is very unlikely that this material will cause a post-transplant rejection. Nevertheless, more studies should be performed on a larger population to confirm its efficacy [5].

Recently it has also been considered the role of debridement with the piezoelectric approach, a new technique that uses a device that generates ultrasonic pulses with microscopic horizontal and vertical oscillations: it is easy to use because it allows the surgeon to have good visibility of the operating field, it is not very bloody and invasive, guaranteeing selectivity for the mineralized bone tissue and therefore greater safety, as it does not cause damage to nerves or other noble anatomical structures in the event of accidental contact with them. Moreover, thanks to the aforementioned qualities, it promotes faster wound healing, is not very painful and brings a better aesthetic result [8].

Given the need for less and less invasive techniques on patients who in most cases have an advanced age, various comorbidities and are susceptible to easily contracting infections or other complications due to the vast extent of the wound (grade 3 osteonecrosis), a recombinant human bone morphogenetic protein-2 has been studied which, used in conjunction with an absorbable collagen sponge (which increases its efficacy by favoring its absorption in the bone for a time sufficient to perform its regenerative function in a complete manner) and with a miniplate (which guarantees structural stability avoiding compression by the surrounding soft tissues), stimulating the action of osteoblasts and osteoclasts contribute to bone reconstruction and rehabilitation over about three months post-surgery without major complications [9].

In conclusion, in order to achieve an optimal result with the surgical approach it is appropriate to make some considerations in this regard: for example, it is essential to acquire images before surgery to verify the extent of the disease, resect the affected margins entirely, combine the surgery with prophylactic antibiotic therapy, avoid the use of prostheses until the osteonecrosis is completely resolved and it would be also safer to withhold the osteoclast inhibitor for a few months before and after the invasive procedures, restarting it when the mucosa has healed [3].

\section{Conclusions}

Although there are various and valid alternatives to treat mandibular osteonecrosis, especially with regard to the more innovative techniques, it would be necessary to carry out further studies on larger populations to confirm their effectiveness. The prognosis would be far more favorable if adequate prevention was carried out, strengthening the education campaign for patients on correct oral hygiene and elimination of risk factors, and approaching the disease in a multidisciplinary manner.

\section{Conflict of interest}

The authors declare the absence of conflict of interest.

\section{References}

1. Foncea C, Von Bischhoffshausen K, Teuber C, Ramírez H, Goñi I, et al. (2020) Osteonecrosis de los maxilares asociada a medicamentos: revisión de la literatura y propuesta para la prevención y manejo. Rev méd Chile 148: 983-991.

2. Singh M, Gonegandla G (2019) Bisphosphonate-Induced Osteonecrosis of the Jaws (BIONJ). J Maxillofac Oral Surg 19: 162-167. [Crossref]

3. https://www.uptodate.com/contents/medication-related-osteonecrosis-of-the-jaw-inpatients-with-cancer\#!

4. Nicolatou-Galitis O, Schiødt M, Mendes R, Ripamonti C, Hope S, et al. (2019) Medicationrelated osteonecrosis of the jaw: definition and best practice for prevention, diagnosis, and treatment. Oral Surg Oral Med Oral Pathol Oral Radiol 127: 117-135. [Crossref]

5. Ragazzo M, Val M, Montagner G, Trojan D, Fusetti S, et al. (2021) Human amniotic membrane: an improvement in the treatment of medication-related osteonecrosis of the jaw (MRONJ)? A case-control study. Cell Tissue Bank. [Crossref]

6. He L, Sun X, Liu Z, Qiu Y, Niu Y (2020) Pathogenesis and multidisciplinary management of medication-related osteonecrosis of the jaw. Int J Oral Sci 12: 30. [Crossref]

7. Ripamonti C, Maniezzo M, Boldini S, Pessi M, Mariani L, et al. (2012) Efficacy and tolerability of medical ozone gas insufflations in patients with osteonecrosis of the jaw treated with bisphosphonates-preliminary data. Medical ozone gas insufflation in treating ONJ lesions. J Bone Oncol 1: 81-87. [Crossref] 
Marasciulo F (2021) The clinical case of an oncological patient affected by mandibular osteonecrosis and literature review about therapeutic alternatives

8. Goker F, Donati G, Grecchi F, Sparaco A, Ghezzi M, et al. (2020) Treatment of BRONJ with ozone/oxygen therapy and debridement with piezoelectric surgery. Eur Rev Med Pharmacol Sci 24: 9094-9103. [Crossref]
9. Kim M, Kim K, Kim B, Kim C, Kim J (2020) Immediate reconstruction of mandibular defect after treatment of medication-related osteonecrosis of the jaw (MRONJ) with rhBMP-2 ACS and miniplate: Review of 3 cases. Int J of Surg Case Rep 66: 25-29. [Crossref]

Copyright: (O2021 Marasciulo F. This is an open-access article distributed under the terms of the Creative Commons Attribution License, which permits unrestricted use, distribution, and reproduction in any medium, provided the original author and source are credited. 